Pacific Journal of Mathematics

THE $\mathscr{F}$-DEPTH OF AN $\mathscr{F}$-PROJECTOR 


\title{
THE $\mathfrak{F}$-DEPTH OF AN $\mathfrak{F}$-PROJECTOR ${ }^{1}$
}

\author{
H. J. SCHMIDT, JR.
}

Let $\mathfrak{F}$ be a saturated formation and let $G$ be a finite solvable group with $\preccurlyeq$-projector $F$. In a fundamental work, Carter and Hawkes have shown that for suitably restricted $\mathfrak{F}$ there is a chain of $\mathscr{F}$-crucial maximal subgroups of $G$ terminating with $F$. It is shown here that the number of links in such a chain is an $\widetilde{f}$-invariant of $G$, called the $\mathfrak{F}$-depth of $F$ in $G$ and written $d_{\Re}(F, G)$.

If $\ell_{\mathfrak{\gamma}}(G)$ is the $\mathfrak{F}$-length of $G$ then, provided $\mathfrak{F}$ is normal subgroup-closed, the inequality $\ell_{\widetilde{F}}(G) \leqq 2 \cdot d_{\mathfrak{F}}(F, G)+1$ is obtained. If $F$ is also nilpotent of nilpotency class $c(F)$, then it is proved that $\ell_{\mathfrak{F}}(G) \leqq d_{\Re}(F, G)+c(F)$.

If $\mathfrak{F}$ and $\mathscr{S}$ are two such suitable saturated formations with $\mathfrak{F} \supseteqq \mathscr{g}$, comparisons of the invariants $d_{\mathfrak{F}}(F, G)$ and $d_{\mathfrak{S}}(H, G)$ are made, where $F$ and $H$ are respectively the $\mathfrak{F}$ - and $\mathfrak{S}$-projectors of the the finite solvable group $G$. In particular, if $H \leqq F$ then $d_{\mathfrak{F}}(F, G) \leqq d_{\mathfrak{F}}(H, G)$, and if in addition $d_{\mathfrak{F}}(F, G)=$ $d_{\mathfrak{q}}(H, G)$ then $H=F$.

1. Introduction. In this paper all groups considered are finite and solvable. Throughout we let $\mathfrak{F}$ be a saturated formation which is locally induced by a class of nonempty, integrated formations $\mathfrak{F}(p)$, one for each prime $p$. The concepts, definitions and notation of this article are included in the above-mentioned paper [2] of Carter and Hawkes. However, we make one standard definition not found there.

DEFINITION 1.1. Let $\mathfrak{F}$ be a nonempty formation and let $G$ be a group. Let $G^{1 \mathfrak{f}}=G^{\mathfrak{s}}$ and define $G^{n \mathfrak{s}}$ recursively by $G^{(n+1) \mathfrak{F}}=\left(G^{n \mathfrak{s}}\right)^{\mathfrak{r}}$. If $\mathfrak{F}$ contains all cyclic groups of prime order then $G^{n \widetilde{S}}=\{1\}$ for some integer $n$. The least such integer $n$ is called the $₹$-length of $G$ and is written $\ell_{\mathfrak{F}}(G)$.

Definition 1.2. Let $S \leqq G$. A factor $H / K$ of $G(H, K \leqq G$ and $K \unlhd H)$ is called an $S$-composition factor of $G$ if $S$ normalizes both $H$ and $K$ and if $H / K$ has no proper, nontrivial subgroup normalized by $S$. A subnormal series $1=H_{0} \triangleleft H_{1} \triangleleft \cdots \triangleleft H_{n}=G$ is called an S-composition series of $G$ if each factor $H_{i+1} / H_{i}$ is an $S$-composition factor of $G$.

The Jordan-Hölder theorem for operator groups ([9], 2.10.2). implies that any two $S$-composition series for $G$ are equivalent. Further,

1 In view of recent work by Schunk, Gaschütz and others, the term $\mathfrak{F}$-projector is used in place of $\mathfrak{F}$-covering subgroup. 
because $G$ is solvable we may infer ([9], 4.4.2 and 4.4.5) that each $S$-composition factor of $G$ is an elementary abelian $p$-group for some prime $p$.

2. The $₹$-depth. In this section we define the notion of the $\mathfrak{F}$-depth of an $\mathfrak{F}$-projector and derive its basic characterization as a "depth".

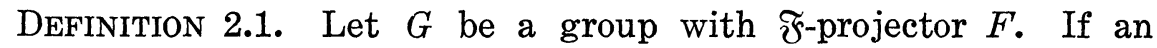
$F$-composition factor $H / K$ of $G$ is a p-group, then $H / K$ is called $F$ central whenever it is centralized by $F^{\Im(p)}$; otherwise it is called $F$ eccentric. If $1=H_{0} \triangleleft H_{1} \triangleleft \cdots \triangleleft H_{n}=G$ is an $F$-composition series for $G$, then the $₹$-depth of $F$ in $G$ is the number of $F$-eccentric factors in this series and is written as $d_{\mathfrak{F}}(F, G)$.

Because the action of $F$ (under conjugation) on equivalent factors is the same and because each two $\mathfrak{F}$-projectors of $G$ are conjugate, it follows that $d_{\mathfrak{f}}(F, G)$ is an invariant of the group $G$ which depends only on the formation $\mathfrak{F}$.

We now establish that all $\mathfrak{F}$-crucial chains from $F$ to $G$ have the same number of links, hence the terminology "depth". The proof given below is due to Trevor Hawkes and is considerably shorter than our original constructive proof.

THEOREM 2.2. Let $G$ be a group with $\widetilde{F}$-projector $F$. Let

$$
F=M_{r}<\cdot M_{r-1}<\cdots<\cdot M_{1}<\cdot G
$$

be an $⿱$ §-crucial chain from $F$ to $G$. Then $d_{\Re}(F, G)=r$.

Proof. Induct on $|G|$. Because $F$ is an $\preccurlyeq$-projector of $M_{1}$ and because the bottom $r-1$ links of the given chain form an $\widetilde{\mho}$-crucial chain from $F$ to $M_{1}$, we have $d_{\mathfrak{F}}\left(F, M_{1}\right)=r-1$.

Suppose that $M_{1}$ is $p$-maximal and put $K=\operatorname{Core}\left(M_{1}\right)$; let $H / K$ be the unique minimal normal subgroup of $G / K$ (see [6], II. 1.4 and II.

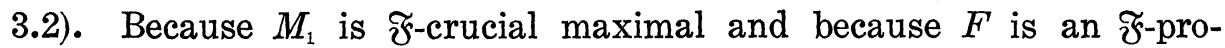
jector of $G$, we have that $G / H \cong M_{1} / K \in \mathfrak{F} \mid \mathfrak{F}(p)$, so that $G=F H$. It follows that $H / K$ is an $F$-eccentric $F$-composition factor of $G$ and that an $F$-composition series of $G$ above $H$ is a chief series of $G$ above $H$ with the $G$-action on these factors equivalent to the $F$-action.

Now let $\mathscr{C}$ be an $F$-composition series of $G$ through $H / K$. The $F$-composition factors in $\mathscr{C}$ below $K$ are $F$-composition factors of $M_{1}$. The observation of the preceding paragraph shows that the factors in $\mathscr{C}$ above $H$ are chief factors of $G$ and because $G / H \in \mathfrak{F}$, each of these factors is $\mathfrak{F}$-central; therefore each is $F$-central since the action of $F$ and $G$ on these factors are equivalent. Hence the isomorphism $G / H \cong$ 
$M_{1} / K$ yields the equality $d_{\mathfrak{\xi}}(F, G)=d_{\widetilde{\S}}\left(F, M_{1}\right)+1=(r-1)+1=r$.

This section concludes with several elementary observations. If $N \unlhd G$ then $F N / N$ is an $\widetilde{f}$-projector of $G / N$. Thus there is an $\widetilde{\mho}$-crucial chain in $G / N$ from $F N / N$ to $G / N$. Because $N$ is contained in the core of any subgroup containing $F N$, we have the following.

Proposition 2.3. If $N \unlhd G$ then there is an $\mathfrak{F}$-crucial chain in $G$ from $F N$ to $G$. The length of this chain is an $\widetilde{\mho}$-invariant of $G$, namely $d_{\Re}(F N / N, G / N)$.

If we write $d_{\tilde{F}}(F N, G)$ for $d_{\mathfrak{F}}(F N / N, G / N)$ then, since $F$ is an $F$-projector of $F N$, the following is immediate.

Proposition 2.4. Let $N \unlhd G$. Then

$$
d_{\Re}(F, G)=d_{\Re}(F, F N)+d_{\Re}(F N, G) \text {. }
$$

3. The Influence of $\widetilde{\wp}$-depth. We now study relations among $d_{\mathfrak{F}}(F, G)$, the $\mathfrak{F}$-length $\ell_{\mathfrak{F}}(G)$ of $G$, and, in case $F$ is nilpotent, the nilpotency class $c(F)$ of $F$. For the first result only, we make the additional assumption that $\mathfrak{F}$ is normal subgroup-closed.

THEOREM 3.1. Let $\mathfrak{F}$ be normal subgroup-closed, and let $G$ be a group with $\mathfrak{\xi}$-projector $F$. Then

$$
\iota_{\mathfrak{F}}(G) \leqq 2 \cdot d_{\mathfrak{F}}(F, G)+1
$$

Proof. Let $r=d_{\Im}(F, G)$ and induct on $|G|$. If $M$ is an $\widetilde{\mho}$-crucial maximal subgroup containing $F$, then $d_{\Re}(F, M)=r-1$. Since $M<$ $G$ we may write $\ell_{\wp}(M) \leqq 2(r-1)+1=2 r-1$.

Let $K=$ Core $(M)$ and, as in the proof of (2.2), let $H / K$ be the unique minimal normal subgroup of $G / K$. Since $M$ is $§$-crucial we have that $M / K \cong G / H \in \mathfrak{F}$ and that $H / K$ is $\mathfrak{F}$-eccentric.

Because $M / K \in \mathfrak{F}$ we obtain $M^{\Im} \leqq K$. But $K / M^{\Im} \unlhd M / M^{\Im} \in \mathfrak{F}$ and $\mathfrak{F}$ is normal subgroup-closed, so that $K^{\Re} \leqq M^{\mathfrak{r}}$. A standard induction argument now shows that $K^{n \Im} \leqq M^{n \Im}$ for all $n$. Therefore $\iota_{\Im}(K) \leqq \iota_{\Im}(M) \leqq$ $2 r-1$. Finally $G / H \in \mathfrak{F}$ and $H / K$ is elementary abelian so that $\iota_{\Im}(G) \leqq \iota_{\Im}(G / K)+\iota_{\Im}(K) \leqq 2+(2 r-1)=2 r+1$, the desired inequality.

We remark that if $\mathfrak{R}$ is the class of nilpotent groups and $G$ is the symmetric group on four letters, then the 2-Sylow subgroups of $G$ are the $\mathfrak{N}$-projectors of $G$ and are maximal. Since $G$ has nilpotent length 3 , the inequality of (3.1) is best possible.

We are grateful to Trevor Hawkes for the following example which shows that the normal subgroup-closed hypothesis of (3.1) is necessary. 
EXAMPLE 3.2. Let $H$ be the subgroup of $\mathrm{SL}(2,11)$ of order 120 which corresponds to the alternating group on five letters under the canonical epimorphism from $\mathrm{SL}(2,11)$ to $\operatorname{PSL}(2,11)$. It is known that $H$ acts fixed point freely on an elementary abelian group $V$ of order $11^{2}([6], p .500)$. The normalizer $K$ of a 2-Sylow subgroup of $H$ has order 24. Let $R$ be the split extension $V K$. Let $W$ be a faithful, irreducible $Z_{5} R$-module and let $G$ be the split extension $W R$. Let $Q$ be the 2-Sylow subgroup of $K$ and let $P$ be a 3-Sylow subgroup of $K$. It is well-known that $Q$ is a quaternion group and that its central involution $z$ is centralized by the generator $x$ of $P$.

Let $\widetilde{F}$ be locally induced by the following. If $p \neq 5,11$ let $\mathfrak{F}(p)=$ $\{1\}$. Let $\widetilde{F}(11)$ be the smallest formation containing $Z_{6}$. If $\mathfrak{S}$ is the formation of all $\{2,3,11\}$-groups in which the 2 -chief factors are central and the nontrivial 11-chief factors are eccentric, then let $\mathfrak{F}(5)$ be the formation of extensions of 5 -groups by groups in $\mathfrak{S}$. Then each $\mathfrak{F}(p)$ is integrated. However, $\widetilde{F}(5)$ is not normal subgroup-closed, for $V \triangleleft$ $V\langle x, z\rangle \in \mathfrak{F}(5)$ and $V$ is an 11-group. A result of Doerk ([3], 2.2) shows that $\mathfrak{F}$ is not normal subgroup-closed.

Finally, $G$ has $\mathfrak{F}$-length 4 and the $\mathfrak{F}$-subgroup $F=W V\langle x, z\rangle$ is maximal. Hence $d_{\widetilde{v}}(F, G)=1$ and the inequality of (3.1) fails for this $G$ and $\mathfrak{F}$.

By way of contrast, we give an example of a group $G$ of $\Re$ length 2 which has a Carter subgroup $A$ of $\mathfrak{N}$-depth $n$, where $n$ is an arbitrary integer.

EXAMPLE 3.3. Let $2<p_{1}<p_{2}<\cdots<p_{n}$ be prime numbers and set $H=Z_{p_{1}} \times Z_{p_{2}} \times \cdots \times Z_{p_{n}}$. For $i=1, \cdots, n$ let $B_{i}=\operatorname{Aut}\left(Z_{p_{i}}\right) \cong$ $Z_{p_{i}-1}$. Set $A=B_{1} \times B_{2} \times \cdots \times B_{n}$ and let $A$ act on $H$ coordinatewise. Put $G=H A$, the split extension. Then $A=C(A)=N(A)$ is nilpotent, so that $A$ is a Carter subgroup of $G$. If $M_{i}=\left(Z_{p_{1}} \times \cdots \times\right.$ $\left.Z_{p_{i}} \times\{1\} \times \cdots \times\{1\}\right) A$, where the factor $\{1\}$ appears $n-i$ times, then $A<\cdot M_{1}<\cdot M_{2}<\cdots<\cdot M_{n-1}<\cdot M_{n}=G$ is an $\mathfrak{U}$-crucial chain of length $n$, so that $d_{3}(A, G)=n$. It is clear that $G$ has nilpotent length 2.

The next lemma is the primary inductive tool for the remainder of this section. We make it as general as possible.

Lemma 3.4. Let $\mathfrak{S}$ be a class of groups which is closed under homomorphic images; and let $f$ be a positive integervalued set function, defined on the class of groups, satisfying

$(\#) \quad f(R) \geqq f(R \theta)$, where $R$ is a group and $\theta$ is an epimorphism of $R$.

Let $G$ be a group in $\mathfrak{S}$ of minimal order which fails to satisfy

$\left(^{*}\right) \quad \ell_{\mathfrak{\gamma}}(G) \leqq d_{\mathfrak{\gamma}}(F, G)+f(F)$, where $F$ is an $\mathfrak{F}$-projector of $G$. 


\section{Then}

(a) $G$ has a unique minimal normal subgroup $A$,

(b) $A$ is complemented and $A=C(A)$,

(c) $A \leqq F$, and

(d) $\ell_{\mathfrak{\gamma}}(G / A)=\ell_{\Im}(G)-1$.

If, in addition, $F$ is nilpotent, then

(e) $F$ is a p-Sylow subgroup of $G$, where $p$ is the exponent of $A$.

Proof. Let $n=\ell_{\mathfrak{F}}(G)$. It is clear that $G \notin \mathfrak{F}$; otherwise $\ell_{\mathfrak{F}}(G)=$ $1, d_{\mathfrak{F}}(F, G)=0$ and $\left({ }^{*}\right)$ obtains since $f(F) \geqq 1$. Therefore $n \geqq 2$.

Let $\mathscr{R}$ be the class of groups of $\preccurlyeq$-length at most $n-1$. It is well-known ([4], 4.3) that $\mathscr{R}$ is a saturated formation.

Let $A$ be a minimal normal subgroup of $G$. Since $G / A \in \mathfrak{F}$ and $F A / A$ is an $₹$-projector of $G / A$, it follows that

( i ) $\quad d_{\Re}(F, G) \geqq d_{\mathfrak{F}}(F A, G) \geqq \ell_{\Im}(G / A)-f(F A / A) \geqq \ell_{\mathfrak{F}}(G / A)-f(F)$. If $\ell_{\mathfrak{\gamma}}(G / A)=n$, then $\left(^{*}\right)$ holds. Therefore $\ell_{\mathfrak{F}}(G / A) \leqq n-1$. Since $\mathscr{R}$ is a (saturated) formation and $\ell_{\Im}(G)=n$, it follows that $A$ is the unique minimal normal subgroup of $G$. Also $A \in \mathfrak{F}$, so that $\ell_{\mathfrak{F}}(G / A)=$ $n-1$ and $A=G^{(n-1) \mathfrak{F}}$. This proves (a) and (d).

If any of the inequalities in (i) is strict, then

$$
d_{\Im}(F, G) \geqq(n-1)-f(F)+1=n-f(F),
$$

contrary to the choice of $G$. Therefore $d_{\mathfrak{x}}(F, G)=(n-1)-f(F)$, whereupon $F=F A$ and $A \leqq F$. This proves (c).

If $M$ is an $\mathscr{R}$-projector of $G$, then $G=M A, M \cap A=1$, and $M$ is maximal. Thus $A=C(A)$, proving (b).

Finally, suppose that $F$ is nilpotent and let $A$ be a $p$-group. Because $A \leqq F$, a nontrivial $p$-complement of $F$ would centralize $A$, contrary to (b). Hence $F$ is a $p$-group. As $\mathfrak{F} \supseteqq \mathfrak{R}$, it is elementary that $N(F)=F$, consequently $F$ must be a $p$-Sylow subgroup of $G$.

Next we establish one of the main results of this section.

THEOREM 3.5. If $F$ is nilpotent, then

$$
\ell_{\S}(G) \leqq d_{\Im}(F, G)+c(F),
$$

where $c(F)$ is the nilpotency class fo $F$.

Proof. If a group $R$ is not nilpotent, we extend the function $c$ by defining $c(R)=|R|$. Then the function $c$ satisfies (\#) of (3.4). Let $G$ be a minimal counter-example to the theorem and let $n=\ell_{\Im}(G)$. Using (3.4) we see that $G$ has a unique minimal normal subgroup $A$ with $A=C(A), A \leqq F, \ell_{\S}(G / A)=n-1$, and that $F$ is a $p$-Sylow subgroup of $G$, where $A$ is a $p$-group. 
Since $Z(F) \leqq C(A)=A$ we have that $c(F / A)<c\left(F^{\prime}\right)$. By the choice of $G$,

$$
d_{\mathfrak{\delta}}(F, G)=d_{\widetilde{\gamma}}(F / A, G / A) \geqq \ell_{\widetilde{\delta}}(G / A)-c(F / A)>(n-1)-c(F) .
$$

Therefore $d_{\Re}(F, G) \geqq(n-1)-c(F)+1=n-c(F)$, contrary to the choice $G$. This proves (3.5).

As a special case of (3.5), we have

COROLLARY 3.6. $\iota_{\Re}(G) \leqq d_{\Re}(C, G)+c(C)$, where $C$ is a Carter subgroup of the group $G$.

In the remainder of this section we give sufficient conditions for the inequality $\iota_{\Re}(G) \leqq d_{\Re}(F, G)+1$ to obtain.

A group $G$ is said to belong to $\mathscr{V}_{\mathfrak{F}}$ [3] provided the set of $\mathfrak{F}$ projectors of $G$ coincides with the set of $\widetilde{\wp}$-normalizers of $G$. Although Doerk [3] has studied $\mathscr{Y}_{\mathfrak{r}}$ in detail, we need only the elementary fact that if $G \in \mathscr{Y}_{\mathscr{F}}$ and $N \unlhd G$ then $G / N \in \mathscr{Y}_{\mathscr{f}}$.

\section{THeOREM 3.7. The inequality}

$$
\ell_{\mathfrak{F}}(G) \leqq d_{\tilde{\mho}}(F, G)+1
$$

obtains provided one of the following holds:

(a) $G$ belongs to $\mathscr{Y}_{\Im}$, or

(b) $F$ complements $G^{\text {r }}$.

Proof. The function $f \equiv 1$ satisfies (\#) of (3.4). Let $\mathfrak{S}$ be the class of groups satisfying either (a) or (b). Note that both hypotheses are invariant under homomorphisms.

Let $G$ be a group of minimal order belonging to $\mathfrak{S}$ and failing to satisfy $(*)$. From (3.4) we infer that $G$ has a unique minimal normal subgroup $A$ with $A=C(A), A \leqq F$, and $\ell_{\S}(G / A)=\ell_{\S}(G)-1$.

Suppose that (a) holds. Since $F$ is an $₹$-normalizer of $G$ and $A \leqq$ $F$, it follows that $A$ is $\widetilde{F}$-central. If $A$ is a p-group then $G / C(A)=$ $G / A \in \mathfrak{F}(p) \subseteq \mathfrak{F}$; therefore $\ell_{\mathfrak{F}}(G)=1$ and $G \in \mathfrak{F}$. But then it is easy to see that $\left(^{*}\right)$ would hold, contrary to the choice of $G$.

Suppose that (b) holds. Since $1<A \cap G^{\mathfrak{f}} \unlhd G$, it follows that $A \leqq G^{\mathfrak{r}}$. Thus $A \leqq F \cap G^{\mathfrak{F}}=1$, a contradiction. This completes the proof.

We now restrict our attention to the class $\mathfrak{N}$ of nilpotent groups and obtain further conditions which afford $\left({ }^{*}\right)$ of (3.7) with $₹$ replaced by $\mathfrak{R}$. For the remainder of this section, $G$ is a group with Carter 
subgroup $C$.

A subgroup $H$ of $G$ is called pronormal provided, for every $x \in G$, there is a $y \in\left\langle H, H^{x}\right\rangle$ with $H^{x}=H^{y}$. Rose shows ([8], 1.6) that if $H$ is pronormal in $G$ then $N(H)$ is the subnormalizer of $H$ in $G$. Secondly, he shows that the class of groups with pronormal system normalizers is a formation ([8], 3.4) which contains (1) the class of $A$-groups (abelian Sylow subgroups), and (2) the class of groups of nilpotent length at most 2.

THEOREM 3.8. The inequality

$$
\iota_{\Re}(G) \leqq d_{\Re}(C, G)+1
$$

obtains provided one of the following holds:

(a) $G$ has p-length 1 for all primes, $p\left|G^{\mathfrak{N}}\right|$, or

(b) $G$ has pronormal system normalizers.

Proof. Again, let $f \equiv 1$ and let $\mathfrak{F}$ be the class of groups satisfying either (a) or (b). It is immediate that $\mathfrak{S}$ is closed under homomorphic images.

Let $G$ be a group of minimal order which belongs to $\mathscr{F}$ and fails to satisfy $(*)$. Using (3.4) we find that $G$ has a unique minimal normal subgroup $A$ of exponent $p$ with $A=C(A), A \leqq C, \ell_{\mathfrak{n}}(G / A)=$ $\iota_{n}(G)-1$, and that $C$ is a $p$-Sylow subgroup of $G$.

Suppose that (a) holds. Because $A$ is the unique minimal normal subgroup of $G$, we infer that $O_{p \prime}(G)=1$. Since $A \leqq G^{\Re}$, we have that $G$ has $p$-length 1 . Thus $G$ has a normal $p$-Sylow subgroup, namely $C$. But this is contrary to $C=N(C)$, so that (a) cannot hold.

Suppose that (b) holds. Observe that $A$ is $\Re$-eccentric because $\iota_{\mathfrak{n}}(G / A)=\iota_{\mathfrak{n}}(G)-1$. Therefore a system normalizer $D$ of $G$ avoids $A$. If $D \leqq C$ then $D$ is both subnormal and pronormal in $C$, whereupon $D$ is normalized by $C([8], 1.5)$. But then $[A, D] \leqq A \cap D=1$, contrary to $A=C(A)$. This completes the proof.

4. $\widetilde{\mho}$-depth versus $\mathfrak{S}$-depth. In this section we consider two formations $\mathfrak{F}$ and $\mathfrak{S}$ which are locally induced by nonempty, integrated formations $\{\mathfrak{F}(p)\}$ and $\{\mathfrak{S}(p)\}$ respectively. We call a local formation $\mathfrak{F}(p)$ full if $\mathfrak{F}(p)=\mathbb{S}_{p} \mathfrak{F}(p)$, where $\mathbb{B}_{p}$ is the class of $p$-groups. The discussion on p. 350-1 of [3] shows that the formations $\mathscr{F}$ and $\mathfrak{S}$ studied here can always be induced by full local formations. Throughout this section we assume that $\mathfrak{S} \subseteq \mathfrak{F}$ and that all local formations for both $\mathfrak{F}$ and $\mathfrak{S}$ are full; it follows readily from this that $\mathfrak{S}(p) \subseteq$ $\mathfrak{F}(p)$ for each prime $p$. In this setting we compare the invariants 
$d_{\mathfrak{F}}(F, G)$ and $d_{\mathfrak{F}}(H, G)$, where $G$ is a group with $\mathfrak{F}$-projector $F$ and Se-projector $H$.

\section{THEOREM 4.1. If $H \leqq F$ then $d_{\mathfrak{F}}(F, G) \leqq d_{\mathfrak{F}}(H, G)$.}

The referee has observed that this theorem follows immediately from the following more general result which we will need later.

THEOREM 4.2. If $H \leqq F$ and $N \unlhd G$, then $d_{\mathfrak{F}}(F, F N) \leqq d_{\mathfrak{5}}(H, H N)$.

Proof. Let $\mathscr{C}$ be an $F$-composition series for $N$. Then $\mathscr{C}$ is an $H$-invariant series and can be refined to an $H$-composition series for $N$. From the definition of the depth function it will suffice to show that each $F$-eccentric factor in $\mathscr{C}$ contains at least one $H$-ecentric composition factor. Suppose then that $M / N$ is $F$-eccentric factor in $\mathscr{C}$. If $M / N$ is $H$-hypercentral then $H M / N \in \mathfrak{F}$. Since $H$ is an $\mathfrak{S}$ projector of $G, H N / N$ is an 5 -projector of $H M / N$, and consequently $H$ covers the factor $M / N$. Thus $F$ covers $M / N$, contradicting the fact that $M / N$ is $F$-eccentric. This completes the proof of (4.2).

Even though $\mathfrak{F} \supseteqq \mathfrak{S}$ it is generally the case that an $\mathfrak{\wp}$-projector does not contain an $\mathfrak{S}$-projector. In fact, if $H$ is contained in no conjugate of $F$, then the inequality of (4.1) can fail as the following example shows.

EXAMPLE 4.3. Let $R$ be the primitive solvable permutation group of degree 8 and order 168. Let $E$ be the 2-Sylow subgroup of $R$, let $P$ be a 7-Sylow subgroup of $R$ and let $Q$ be a 3-Sylow subgroup of $R$ which normalizes $P$. Let $W$ be a faithful, irreducible $Z_{5} R$-module and let $V$ be a faithful, irreducible $Z_{11} W R$-module, where $W R$ is the split extension. Let $G$ be the split extension of $V$ by $W R$. Finally let $H=V W E Q$ and $M=V E P Q$. It follows that $H$ and $M$ are maximal subgroups of $G$ of indices 7 and $|W|$ respectively.

If $\pi$ is a set of primes, let $\mathbb{S}_{\pi}$ be the set of $\pi$-groups. Define $\mathfrak{K}$ and $\mathfrak{F}$ locally as follows:

$$
\begin{aligned}
& \mathfrak{S}(2)=\mathfrak{F}(2)=\mathbb{G}_{\{2,3,7\}} \text { and } \mathfrak{S}(5)=\mathfrak{F}(5)=\mathbb{C}_{\{2,3,5\}} \\
& \mathscr{S}(7)=\mathbb{S}_{\{7\}} \\
& \mathfrak{F}(7)=\mathbb{S}_{\{7\}} \mathfrak{A}_{6} \text {, where } \mathfrak{A}_{6} \text { is the formation of abelian groups } \\
& \text { of exponent dividing } 6 \\
& \mathfrak{S}(11)=\mathfrak{F}(11)=\mathbb{S}_{\{11\}} \mathscr{L} \text {, where } \mathscr{L}=\{G: G / F(G) \text { has order } \\
& \text { prime to } 7\} \text {. }
\end{aligned}
$$

For $p \neq 2,5,7,11$ let $\mathfrak{S}(p)=\mathfrak{F}(p)=\mathbb{S S}_{p}$. Then $\mathfrak{F}$ and $\mathfrak{F}$ satisfy the general conditions imposed in this section, and $\mathfrak{F} \neq \mathfrak{F}$ since $P Q \in \mathfrak{F} \mid \mathfrak{S}$. 
It follows that $H$ is an $\mathscr{S}_{2}$-projector of $G$; thus $d_{\mathfrak{\$}}(H, G)=1$. Fur-

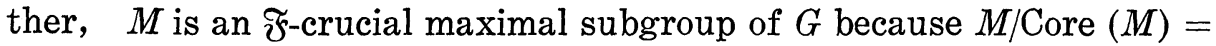
$M / V \cong E P Q \in \mathfrak{F} \mid \mathfrak{F}(5)$. Hence $M$ contains an $\mathfrak{F}$-projector of $G$ ([2], 5.4). If $L$ is the largest normal 11-nilpotent subgroup of $M$, then $M / L=M / V \cong E P Q \notin \mathfrak{F}(11)$, so that $M \notin \mathfrak{F}$ ([2], 2.5). It now follows that $M$ must contain an $\widetilde{F}$-projector $F$ of $G$ as a proper subgroup. Therefore $d_{\mathfrak{f}}(F, G) \geqq 2>1=d_{\mathfrak{5}}(H, G)$. It is obvious that $F$ does not contain a conjugate of $H$.

We give still another example to demonstrate the complex relationship between $\mathfrak{F}$-depth and $\mathfrak{S}$-depth. In particular, we display two chains of $\preccurlyeq$-abnormal maximal subgroups of different lengths which terminate with a given Carter subgroup.

EXAMPLE 4.4. Let $\mathfrak{\mho}$ be the class of 3-nilpotent groups and let $\mathfrak{S}=\mathfrak{N}$. Then $\mathfrak{R}$ can be induced by the local formations $\mathfrak{N}(p)=\mathbb{S}_{p}$, for each prime $p$, and $\mathfrak{F}$ by the local formations $\mathfrak{F}(3)=\mathbb{S}_{3}$ and $\mathfrak{F}(p)=$ $\mathfrak{F}$ for $p \neq 3$.

Let $G$ be the group of all semi-linear transformations over $G F\left(3^{3}\right)$ of the form $x \mapsto a x^{t}+b$, where $a, b \in G F\left(3^{3}\right)$ with $a \neq 0$ and where $t$ belongs to the Galois group of $G F\left(3^{3}\right)$ over its prime field. Then $|G|=27 \cdot 26 \cdot 3$. Let $F$ be those transformations in which $b=0, C$ those in which $b=0$ and $a= \pm 1, P$ those in which $a=1$, and $A$ those in which $a=1$ and $t=1$.

Certainly $F$ is 3-nilpotent and maximal in $G$, hence $F$ is an $\mathfrak{F}$ projector of $G$ and $d_{\widetilde{x}}(F, G)=1$. Also $C<\cdot F$ because $|F: C|=13$. Since $P$ is the 3-Sylow subgroup of $G$ it has a central series $1 \triangleleft B_{1} \triangleleft$ $B_{2} \triangleleft A \triangleleft P$ with factors of order 3 ; consequently the series $1 \triangleleft B_{1} \triangleleft$ $B_{2} \triangleleft A$ is $C$-invariant. Because the Galois group fixes the prime field elementwise (here identified with $a= \pm 1$ ), $C$ is nilpotent. It now follows that $C<\cdot C B_{1}<\cdot C B_{2}<\cdot C A<\cdot G$ is an $\Re$-crucial chain in $G$. Therefore $C$ contains a Carter subgroup of $G$ ([2], 5.4). However, since $C$ is nilpotent it must be a Carter subgroup of $G$.

Finally, note the numerical relations: $d_{\mathfrak{n}}(C, G)=4, d_{\mathfrak{n}}(C, F)=1$ and $d_{\Re}(F, G)=1$. In particular, $d_{\Re}(C, F)+d_{\tilde{\mho}}(F, G) \nsim d_{\Re}(C, G)$.

In the previous example it was seen that the equality $d_{\mathfrak{\xi}}(H, G)=$ $d_{\mathfrak{5}}(H, F)+d_{\Re}(F, G)$ failed even though $H \leqq F$. We now give a rather strong sufficient condition for this equality to obtain.

THEOREM 4.5. If $G \in \mathfrak{N S}$ then

(a) $H$ is contained in a conjugate of $F$, and

(b) $d_{\mathfrak{\xi}}(H, G)=d_{\mathfrak{\xi}}(H, F)+d_{\mathfrak{\Im}}(F, G)$.

Proof. Note that $G$ also belongs to $\mathfrak{R} \mathfrak{F}$ since $\mathfrak{S} \subseteq \mathfrak{F}$.

(a) Every $\mathfrak{F}$-crucial link above $F$ is $\mathfrak{S}$-abnormal since $\mathfrak{S}(p) \subseteq$ 
$\mathfrak{F}(p)$ for each prime $p$; thus $([2], 4.2)$ implies that $F$ contains an $\mathfrak{S}$-normalizer of $G$. As $G \in \mathfrak{N S S}$ it follows that $H$ is an $\mathfrak{S}$-normalizer of $G$, whereupon $H$ is contained in a conjugate of $F$.

(b) Consider an $\preccurlyeq$-crucial chain from $F$ to $G$. Again, because $\mathfrak{S}(p) \subseteq \mathfrak{F}(p)$ for each prime $p$, each $\mathfrak{F}$-crucial link is $\mathfrak{S}$-critical. Because

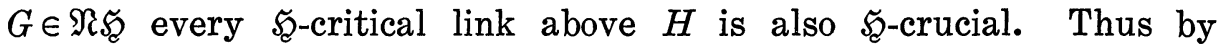
adjoining an $\mathfrak{\mho}$-crucial chain from $F$ to $G$ to an $\mathscr{S}$-crucial chain from

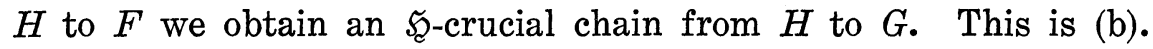

Since the derived group of a supersolvable group is nilpotent, we have

COROLLARY 4.6. If $G$ is supersolvable, then

(a) $H$ is contained in a conjugate of $F$, and

(b) $\quad d_{\mathfrak{f}}(H, G)=d_{\mathfrak{\Phi}}(H, F)+d_{\mathfrak{F}}(F, G)$.

THEOREM 4.7. If $H \leqq F$ and $d_{\mathfrak{5}}(H, G)=d_{\mathfrak{F}}(F, G)$, then $H=F$.

We remark that if (b) of (4.5) held for all groups $G$, then a proof of (4.7) would proceed as follows. Combine the equalities $d_{\mathfrak{5}}(H, G)=$ $d_{\mathfrak{F}}(F, G)$ and $d_{\mathfrak{5}}(H, G)=d_{\mathfrak{ई}}(H, F)+d_{\mathfrak{F}}(F, G)$ to obtain that $d_{\mathfrak{\xi}}(H$, $F)=0$, and hence infer that $H=F$. Because the restrictions on $G$ in (4.5) are quite strong, it is surprising that (4.7) holds in general.

Proof of (4.7). Let $G$ be a minimal counter-example and let $A$ be a minimal normal subgroup of $G$. Then $H A \leqq F A$ and (4.2) implies that $d_{\mathfrak{\Im}}(F, F A) \leqq d_{\mathfrak{F}}(H, H A)$. Since $d_{\mathfrak{F}}(F, G)=d_{\mathfrak{F}}(H, G)$, the equality $d_{\mathfrak{F}}(F, F A)+d_{\mathfrak{\Im}}(F A, G)=d_{\mathfrak{5}}(H, H A)+d_{\mathfrak{5}}(H A, G)$ and (4.1) imply that $d_{\mathfrak{f}}(F, F A)=d_{\mathfrak{\xi}}(H, H A)$ and $d_{\mathfrak{ई}}(H A, G)=d_{\mathfrak{\Im}}(F A, G)$. By the choice of $G$ we can conclude that $F A=H A$.

If $A \leqq F$, then $d_{\mathfrak{F}}(F, F A)=0$, so that $d_{\mathfrak{F}}(H, H A)=0$; hence $A \leqq$ $H$. But then, $F=F A=H A=H$, a contradiction. Therefore $A \leqq F$. If $F A<G$, then because of the choice of $G$, we have that $F=$ $H$ since $d_{\tilde{f}}(H, H A)=d_{\mathfrak{\vartheta}}(F, F A)$. Thus $G=F A$ and $F \cap A=1$. But then $F=F \cap F A=F \cap H A=H(F \cap A)=H$, the final contradiction.

\section{REFERENCES}

1. R. W. Carter, Nilpotent self-normalizing subgroups and system normalizers, Proc. London Math. Soc., 12 (1962), 535-563.

2. R. W. Carter and T. Hawkes, The $\mathfrak{F - n o r m a l i z e r s ~ o f ~ a ~ f i n i t e ~ s o l v a b l e ~ g r o u p , ~ J . ~ A l g e b r a , ~}$ 5 (1967), 175-202.

3. K. Doerk, Zur Theorie der Formationen endlicher aufläsbarer Gruppen, J. Algebra, 13 (1969), 345-373.

4. B. Fischer, Classes of conjugate subgroups in finite solvable groups, Yale University lecture notes, (1966).

5. D. Gorenstein, Finite Groups, New York, Evanston, London: Harper and Row, 1968. 
6. B. Huppert, Endliche Gruppen I, Berlin, Heidelberg, New York: Springer, 1967. 7. - Zur Theorie der Formationen, Arch. Math., 19 (1968), 561-574.

8. J. S. Rose, Finite solvable groups with pronormal system normalizers, Proc. London Math. Soc., 17 (1967), 447-467.

9. W. R. Scott, Group Theory, Englewood Cliffs, New Jersey: Prentice-Hall, 1964.

Received November 1, 1971 and in revised form December 6, 1971. This paper was prepared as part of a doctoral dissertation presented to the University of Oregon. The author thanks Professor Charles R. B. Wright for his guidance and advice during its preparation. The author also thanks Gary M. Seitz for several suggestions.

ILLINOIS STATE UNIVERSITY 



\section{PACIFIC JOURNAL OF MATHEMATICS}

\section{EDITORS}

D. Gilbarg and J. Milgram

Stanford University

Stanford, California 94305

\author{
R. A. Beaumont \\ University of Washington \\ Seattle, Washington 98105
}

J. DuGUNDJI

Department of Mathematics

University of Southern California

Los Angeles, California 90007

Richard ARENS

University of California

Los Angeles, California 90024

\section{ASSOCIATE EDITORS}
E. F. BECKENBACH
B. H. NeumanN
F. WOLF
K. YosHIDA

\section{SUPPORTING INSTITUTIONS}

UNIVERSITY OF BRITISH COLUMBIA

CALIFORNIA INSTITUTE OF TECHNOLOGY

UNIVERSITY OF CALIFORNIA

MONTANA STATE UNIVERSITY

UNIVERSITY OF NEVADA

NEW MEXICO STATE UNIVERSITY

OREGON STATE UNIVERSITY

UNIVERSITY OF OREGON

OSAKA UNIVERSITY

\author{
UNIVERSITY OF SOUTHERN CALIFORNIA \\ STANFORD UNIVERSITY \\ UNIVERSITY OF TOKYO \\ UNIVERSITY OF UTAH \\ WASHINGTON STATE UNIVERSITY \\ UNIVERSITY OF WASHINGTON
* * * *
AMERICAN MATHEMATICAL SOCIETY \\ NAVAL WEAPONS CENTER
}

The Supporting Institutions listed above contribute to the cost of publication of this Journal, but they are not owners or publishers and have no responsibility for its content or policies.

Mathematical papers intended for publication in the Pacific Journal of Mathematics should be in typed form or offset-reproduced, (not dittoed), double spaced with large margins. Underline Greek letters in red, German in green, and script in blue. The first paragraph or two must be capable of being used separately as a synopsis of the entire paper. The editorial "we" must not be used in the synopsis, and items of the bibliography should not be cited there unless absolutely necessary, in which case they must be identified by author and Journal, rather than by item number. Manuscripts, in duplicate if possible, may be sent to any one of the four editors. Please classify according to the scheme of Math. Rev. Index to Vol. 39. All other communications to the editors should be addressed to the managing editor, Richard Arens, University of California, Los Angeles, California, 90024.

50 reprints are provided free for each article; additional copies may be obtained at cost in multiples of 50 .

The Pacific Journal of Mathematics is issued monthly as of January 1966. Regular subscription rate: $\$ 48.00$ a year (6 Vols., 12 issues). Special rate: $\$ 24.00$ a year to individual members of supporting institutions.

Subscriptions, orders for back numbers, and changes of address should be sent to Pacific Journal of Mathematics, 103 Highland Boulevard, Berkeley, California, 94708.

PUBLISHED BY PACIFIC JOURNAL OF MATHEMATICS, A NON-PROFIT CORPORATION

Printed at Kokusai Bunken Insatsusha (International Academic Printing Co., Ltd.), 270, 3-chome Totsuka-cho, Shinjuku-ku, Tokyo 160, Japan. 


\section{Pacific Journal of Mathematics}

\section{Vol. 46, No. 2 December, 1973}

Christopher Allday, Rational Whitehead products and a spectral sequence of

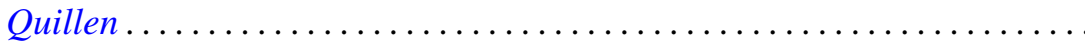

James Edward Arnold, Jr., Attaching Hurewicz fibrations with fiber

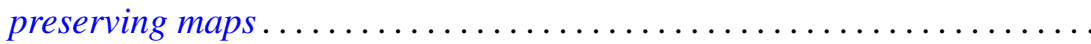

Catherine Bandle and Moshe Marcus, Radial averaging transformations with various metrics.................................

David Wilmot Barnette, A proof of the lower bound conjecture for convex

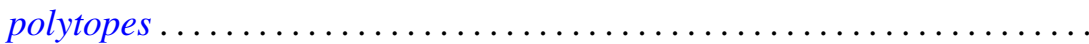

Louis Harvey Blake, Simple extensions of measures and the preservation of

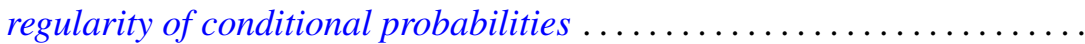

James W. Cannon, New proofs of Bing's approximation theorems for

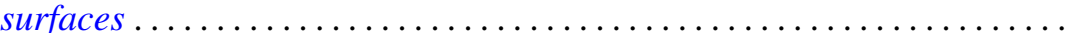

C. D. Feustel and Robert John Gregorac, On realizing HNN groups in

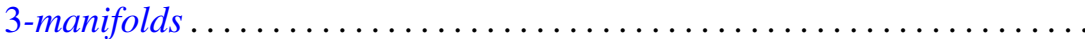

Theodore William Gamelin, Iversen's theorem and fiber algebras . . . . . . . . 389

Daniel H. Gottlieb, The total space of universal fibrations . . . . . . . . . . . .

Yoshimitsu Hasegawa, Integrability theorems for power series expansions of

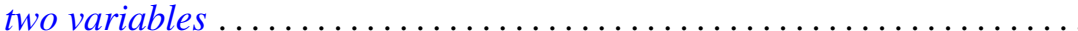

Dean Robert Hickerson, Length of period simple continued fraction expansion of $\sqrt{ } d$

Herbert Meyer Kamowitz, The spectra of endomorphisms of the disc algebra.

Dong S. Kim, Boundedly holomorphic convex domains

Daniel Ralph Lewis, Integral operators on $\mathscr{L}_{p}$-spaces ...

John Eldon Mack, Fields of topological spaces . . . . . . . . .

V. B. Moscatelli, On a problem of completion in bornology

Ellen Elizabeth Reed, Proximity convergence structures. .

Ronald C. Rosier, Dual spaces of certain vector sequence spaces .

Robert A. Rubin, Absolutely torsion-free rings

Leo Sario and Cecilia Wang, Radial quasiharmonic functions . .

James Henry Schmerl, Peano models with many generic classes .

H. J. Schmidt, The $\mathscr{F}$-depth of an $\mathscr{F}$-projector ............

Edward Silverman, Strong quasi-convexity. . . . . . . . . . . . . . . . . 549

Barry Simon, Uniform crossnorms ....................... 555

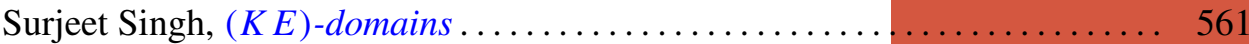

Ted Joe Suffridge, Starlike and convex maps in Banach spaces . . . . . . . . 575

Milton Don Ulmer, $C$-embedded $\Sigma$-spaces . . . . . . . . . . . . . . . . 591

Wolmer Vasconcelos, Conductor, projectivity and injectivity . . . . . . . . . 603 